\title{
Distinguishing between overdrive excited and suppressed ventricular beats in guinea pig ventricular myocardium
}

\author{
Amara Greer-Short ${ }^{1,2}$ and Steven Poelzing ${ }^{1,2 *}$ \\ Department of Biomedical Engineering and Mechanics, Center for Heart and Regenerative Medicine, Virginia Polytechnic Institute and State University, Virginia \\ Tech Carilion Research Institute, Roanoke, VA, USA \\ ${ }^{2}$ School of Biomedical Engineering and Sciences, Virginia Polytechnic Institute and State University, Blacksburg, VA, USA
}

Edited by:

Zhilin Qu, University of California,

Los Angeles, USA

Reviewed by:

Thomas Hund, Ohio State

University, USA

Crystal M. Ripplinger, University of

California, Davis, USA

\section{*Correspondence:}

Steven Poelzing, Virginia Tech

Carilion Research Institute, 2

Riverside Circle, Roanoke, VA

24016, USA

e-mail: poelzing@vtc.vt.edu
Rapid ventricular pacing rates induces two types of beats following pacing cessation: recovery cycle length (RCL) prolongation (overdrive suppression) and RCL shortening (overdrive excitation). The goals of this study were to compare common experimental protocols for studying triggered activity in whole-heart preparations and differentiate between recovery beats using a new methodology. Post-pacing recovery beat cycle length $(R C L)$ and $Q R S$ were normalized to pre-paced R-R and QRS intervals and analyzed using a $\mathrm{K}$-means clustering algorithm. Control hearts only produced suppressed beats: RCL ratio increased with rapid pacing ( $25 \pm 4.0 \%, n=10)$ without changing QRS duration. Rapid pacing during hypercalcemia + hypothermia $\left(5.5 \mathrm{mM}\right.$ and $\left.34^{\circ} \mathrm{C}\right)$ produced significantly earlier excited beats $(53 \pm 14 \%, n=5)$ with wider QRS durations ( $58 \pm 6.3 \%, n=5)$ than suppressed beats. Digoxin + hypothermia $(0.75 \mu \mathrm{M})$ produced the most excited beats with significantly earlier RCL $(44 \pm 3.2 \%, n=6)$ and wider QRS $(60 \pm 3.1 \%, n=6)$ ratios relative to suppressed beats. Increasing pacing further shortened $\mathrm{RCL}$ ( $30 \pm 7.8 \%$, $n=6)$. In a prospective study, TTX $(100 \mathrm{nM})$ increased $\mathrm{RCL}$ ratio $(15 \pm 6.0 \%, n=10)$ without changing the QRS duration of excited beats. The algorithm was compared to a cross-correlation analysis with 93\% sensitivity and 94\% specificity. This ECG based algorithm distinguishes between triggered and automatic activity.

Keywords: automaticity, triggered activity, overdrive excitation, overdrive suppression, hypothermia, digoxin, hypercalcemia

\section{INTRODUCTION}

The heart electrically excites and contracts in a specific order, as automaticity in sino-atrial nodal cells sets a rhythm faster than other cardiac cells. However, at times electrical activity can originate prematurely at ectopic sites, leading to arrhythmias. Early work in canine purkinje fibers using cardiac glycosides found premature low amplitude membrane depolarizations following rapid pacing (Ferrier et al., 1973; Hashimoto and Moe, 1973; Rosen et al., 1973). These small depolarizations grew in amplitude as the pacing rate was increased, and the interval between the last paced beat and the onset of the oscillation decreased (Ferrier et al., 1973; Hashimoto and Moe, 1973). Close observation led to the discovery that the onset of these small depolarizations in Purkinje fibers were concordant with the onset of ventricular arrhythmias (Rosen et al., 1973). This suggested that small depolarizations may be responsible for cardiac glycoside-induced ventricular arrhythmias. Indeed, these depolarizations, later known as delayed afterdepolarizations (DADs), could initiate an action potential if the amplitude was large enough (Ferrier et al., 1973). These action potentials initiated by DADs later became known

Abbreviations: BCL, Basic Cycle Length; DAD, Delayed Afterdepolarization; OE, Overdrive Excitation; OS, Overdrive Suppression; NCX, Sodium-Calcium Exchanger; NKA, Sodium-Potassium ATPase; TTX, Tetrodotoxin; RCL, Recovery Beat Cycle Length; TA, Triggered Activity. as triggered activity (TA), as their initiation was dependent on rapid pacing or "trigger" (Cranefield, 1977). Finally, the recovery beat cycle length of TA decreases as pacing rate increases, and this is referred to as overdrive excited (OE) behavior. In contrast, rapid pacing can also overdrive suppress (OS) the recovery beat by temporarily suppressing normal automaticity (Malfatto et al., 1988; Iinuma et al., 1989), presumably by hyperpolarizing the membrane (Vassalle, 1970). Therefore, rapid pacing can produce mechanistically different recovery beats: normal (OS) and arrhythmogenic (OE).

Later observations in intact heart models discovered that glycosides decreased recovery beat cycle length following rapid pacing (Malfatto et al., 1988; Vos et al., 1990), suggesting that triggered recovery beats in intact models with glycoside toxicity were due to TA, as their behavior was consistent with data from Purkinje fibers. More generally speaking, cardiac glycosides and many other interventions such as hypercalcemia (Plummer et al., 2011), catecholamine perfusion (Priori and Corr, 1990), and myocardial infarction (Lazzara et al., 1973) are associated with an increased incidence of DADs and TA.

Importantly, the recovery beat cycle length may be insufficient to distinguish between $\mathrm{OS}$ and $\mathrm{OE}$ beats. For example, an automatic beat could occur before a triggered rhythm at slow pacing rates and be classified as a triggered rhythm. Therefore, additional criteria are necessary to distinguish between 
mechanistically different recovery beats. The goals of this study were to (1) Differentiate between OS and OE beats with a new methodology, (2) Develop a robust protocol for creating OE beats that can be studied, and (3) Demonstrate that OE beats can be pharmacologically manipulated.

\section{MATERIALS AND METHODS}

The investigation conforms to the Guide for the Care and Use of Laboratory Animals published by the US National Institutes of Health (NIH Publication No. 85-23, revised 1996) and has been approved by Institutional Animal Care and Use Committee (IACUC) at Virginia Polytechnic Institute and State University.

\section{GUINEA PIG LANGENDORFF PREPARATIONS}

Retired breeder male guinea pigs $(n=37)$ were anesthetized with sodium pentobarbital $(325 \mathrm{mg} / \mathrm{kg})$ and injected intraperitoneally with heparin to prevent blood clotting. Atria were excised to remove competitive stimulation from atria, and ventricles were perfused in a Langendorff system with oxygenated Tyrode solution (in $\mathrm{mM}, \mathrm{CaCl}_{2}$ 1.25, $\mathrm{NaCl} 140, \mathrm{KCl} 4.56$, dextrose 5.5, $\mathrm{MgCl}_{2} 0.7$, HEPES $10 ; \mathrm{pH}$ to 7.4 with approximately $5.5 \mathrm{~mL}$ of $\mathrm{NaOH}$ ) at $37^{\circ} \mathrm{C}$ and $50 \mathrm{mmHg}$. Motion was reduced using $7.5 \mathrm{mM}$ 2,3-diacetylmonoxime.

\section{ELECTROCARDIOGRAPHY (ECG) AND INTERVENTIONS}

A volume-conducted bath electrocardiogram (ECG) similar to lead I was continuously recorded to determine recovery cycle length (RCL) and QRS duration of the QRS complex immediately after the cessation of pacing. Hearts were paced with plunge bipolar electrodes fixed in the basal interventricular septum at pacing rates from 200 to $375 \mathrm{bpm}$ for $15 \mathrm{~s}$. Hearts were also perfused with the glycoside digoxin $(0.75 \mu \mathrm{M})$, high extracellular calcium $(5.5 \mathrm{mM} \mathrm{CaCl} 2)$, or the sodium channel inhibitor tetrodotoxin (TTX, $100 \mathrm{nM}$ ). Hearts were maintained at normal $\left(37^{\circ} \mathrm{C}\right)$ and hypothermic $\left(34^{\circ} \mathrm{C}\right)$ temperatures. ECG measurements were made $10 \mathrm{~min}$ after the start of perfusion. RCL and QRS ratios were then normalized to the pre-paced beat's cycle length (BCL) and the QRS, called RCL and QRS ratios, respectively. The recovery window was $1500 \mathrm{~ms}$. A k-means two cluster analysis, which is an automated clustering algorithm that calculates the distance from each data point to a continuously calculated centroid of each cluster, was used to separate the control, hypercalcemic, digoxin, and hypothermic data into two clusters (Cluster 1 and Cluster 2).

A prospective analysis was performed for the digoxin + tetrodotoxin data set, where the centroids of Clusters 1 and 2 from the original data set (control, hypercalcemia, digoxin, hypothermia) were calculated and recovery beats for digoxin + tetrodotoxin were stratified into two clusters depending on the centroid they were closest to.

To determine sensitivity and specificity of our new algorithm, cluster classification was compared to a previously validated cross-correlation analysis as the gold-standard (Kyle et al., 2009). The maximum cross-correlation coefficient was used in this study to analyze QRS morphology similarities between Cluster 1 and 2 recovery beats and their prospective pre-paced beats. True positive was defined as $\mathrm{OE}$ beats with a coefficient $<0.9$, false positive as OE beats with a coefficient $\geq 0.9$, true negative as OS beats with a coefficient $\geq 0.9$, false negative as OS beats with a coefficient $<0.9$.

\section{STATISTICAL ANALYSIS}

Where appropriate, unpaired and paired two-tailed Student's $t$ tests with equal/unequal variance or the nonparametric Wilcoxon rank-sum test were used to analyze data significance. The F-test for equality of two variances was used to analyze variance significance. The Sidak correction for multiple comparisons was used. A $p<0.05$ was considered significant. Values are reported as mean \pm standard error. Retrospective power analyses were performed to determine the recommended sample sizes. The minimum number of samples per intervention was calculated using a power analyses for a 1 sided, two independent sample normal distribution test with alpha 0.05 and a power of 0.8 . Minimum number of beats:

- Hypercalcemia: 5 OS and 5 OE beats

- Hypothermia + hypercalcemia: 5 OS and 5 OE beats

- Digoxin: 4 OS and 4 OE beats

- Hypothermia + digoxin: 5 OS and 5 OE beats

- Digoxin + TTX: 4 OS and 4 OE beats

\section{RESULTS}

\section{OVERDRIVE SUPPRESSION AND EXCITATION}

Example ECGs from control hearts in Figure 1A demonstrate that RCL increases when pacing rate is increased from 200 to 375 bpm. Representative ECGs in Figure 1A also demonstrate in hearts perfused with $0.75 \mu \mathrm{M}$ digoxin that increasing pacing can decrease the recovery beat's RCL. For all experiments, increasing pacing rate in control hearts from 200 to $375 \mathrm{bpm}$ significantly increases RCL, whereas during digoxin, rapid pacing decreases RCL (Figure 1B).

The QRS complexes of the recovery beat in the control hearts in Figure 1A appear similar, while the digoxin recovery beats at 375 bpm exhibit variable ECG characteristics. Specifically during rapid pacing, digoxin recovery beats can be associated with 1. narrow QRS durations and increased RCL (Figure 2, top panel) and with 2 wide QRS durations and decreased RCL (bottom panel) relative to the pre-paced beat. In summary, at $200 \mathrm{bpm}$, the RCL of digoxin recovery beats was $511 \pm 94 \mathrm{~ms}$ and the QRS $27 \pm$ $8 \mathrm{~ms}$. At $375 \mathrm{bpm}$, RCL significantly decreased to $388 \mathrm{~ms}$ but the standard deviation significantly increased to $154 \mathrm{~ms}$. Conversely, QRS increased to $36 \mathrm{~ms}$ without significantly altering standard deviation. This suggests that recovery beats in the digoxin group (Figure 1B) may be composed of beats that could be either automatic or triggered activity (TA). Therefore, it is important to stratify beat type in order to study mechanistic behaviors.

To stratify recovery beats, we used a k-means 2-cluster analysis on raw RCL and QRS values. Figure 3 demonstrates preferential clustering based on RCL values, because the range of RCL values (205 to $1226 \mathrm{~ms}$ ) is significantly larger than the QRS duration range (18 to $64 \mathrm{~ms}$ ). More specifically, k-means is based on each data point's distance from a centroid. Therefore the larger range has a larger effect on clustering, and as a result, clustering raw RCL and QRS reduces the influence of QRS duration. 

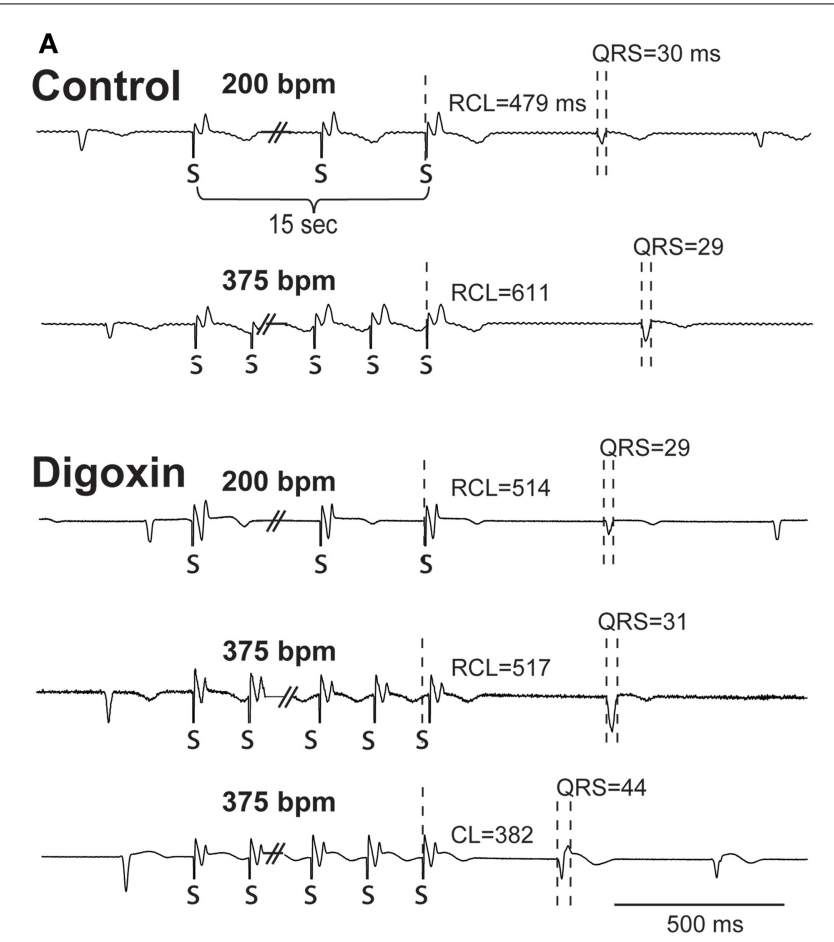

B

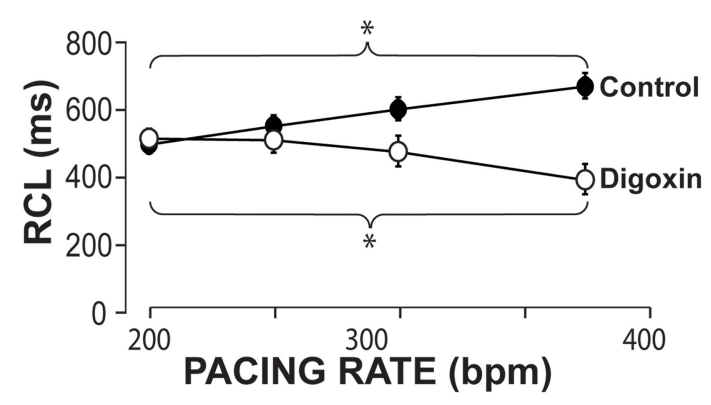

FIGURE 1 | Recovery beats characteristics in response to slow and rapid pacing. (A) Representative volume-conducted electrocardiograms of control (top) and digoxin (bottom) at pacing rates of 200 and $375 \mathrm{bpm}$. Paced beats are marked by "S." (B) Summary data of the recovery beat cycle length following different pacing rates. Control data had an increase in $\mathrm{RCL}\left({ }^{*} p<0.05, n=11\right)$ as pacing rate was increased, while digoxin had a decrease in $\mathrm{RCL}(* p<0.05, n=12)$.

Furthermore, pharmacologic intervention and inter-animal variability can underlie differences in the basic cycle length (BCL) and QRS morphology of native beats, and therefore the recovery beat. For example, any intervention which increased native rhythm rates would likely decrease all recovery beat RCL. This analysis could classify the recovery beat as an OE beat without accounting for the fact that BCL changed. A similar argument can be made for QRS.

To account for BCL and QRS variability, we chose to normalize recovery beat RCL and QRS. In Figure 4A, an ECG before, during, and after pacing illustrates how the BCL and QRS durations were measured prior to pacing (QRS1), and how the RCL and QRS durations were measured post-pacing (QRS2). We normalized the RCL and QRS duration of the recovery beat to the

\section{5 bpm \\ Narrow QRS}
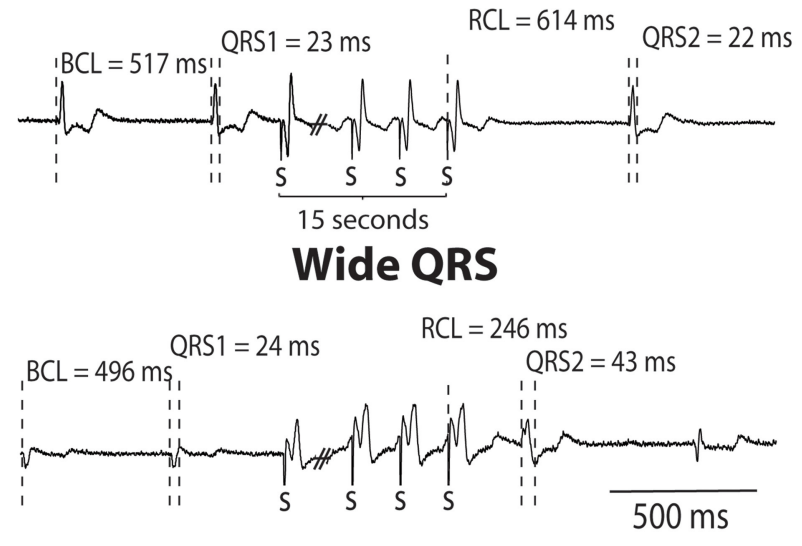

FIGURE 2 | Digoxin recovery beats can manifest a late recovery-narrow QRS duration or an early recovery-wide QRS duration. Representative ECGs from digoxin experiments. Recovery beats with narrow and wide QRS complexes are shown in response to $15 \mathrm{~s}$ of rapid pacing. Figure illustrates representative cycle lengths (CL) and QRS of pre-paced beats, and the cycle length $(\mathrm{RCL})$ and $\mathrm{QRS}$ or the recovery beat.

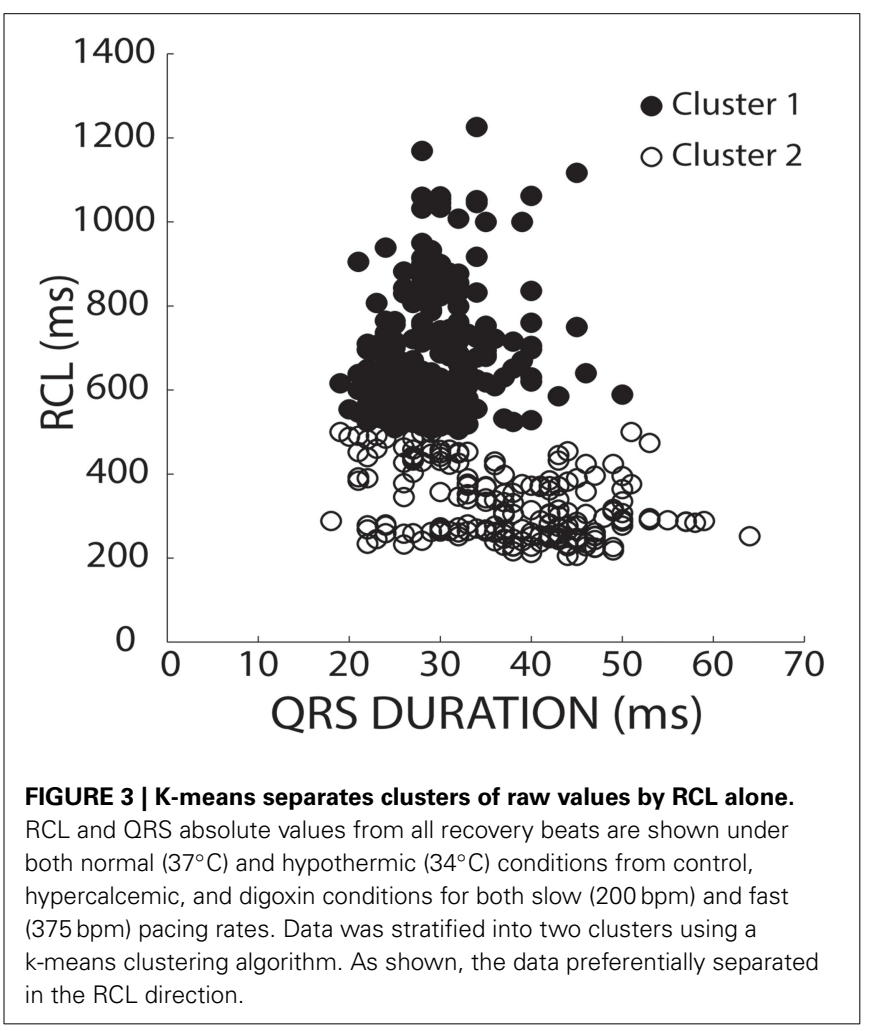

pre-paced beat's BCL and QRS duration, respectively (Figure 4A). The RCL and QRS ratios of all recovery beats from multiple interventions were then plotted and the aforementioned k-means 2-cluster analysis performed. Figure 4B demonstrates recovery beat classification, where Cluster 1 (filled circles) is composed 


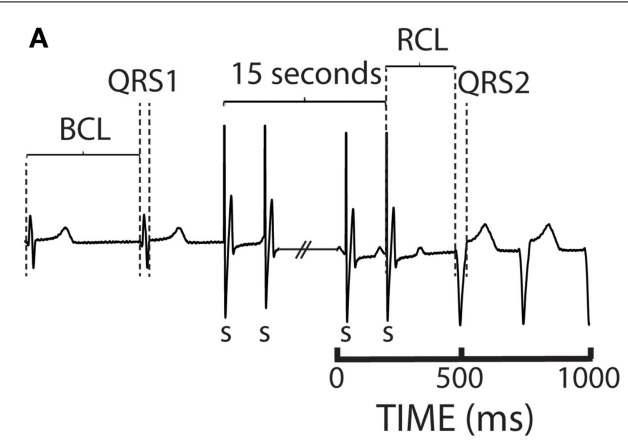

FIGURE 4 | K-means separates ratio values by both parameters. (A) Representative ECGs showing how $\mathrm{RCL}$ and $\mathrm{QRS}$ ratios were quantified. The recovery beat's cycle length (RCL) and QRS (QRS2) were normalized to the $\mathrm{R}-\mathrm{R}$ interval or basic cycle length (BCL) and QRS (QRS1). (B) Shown are the

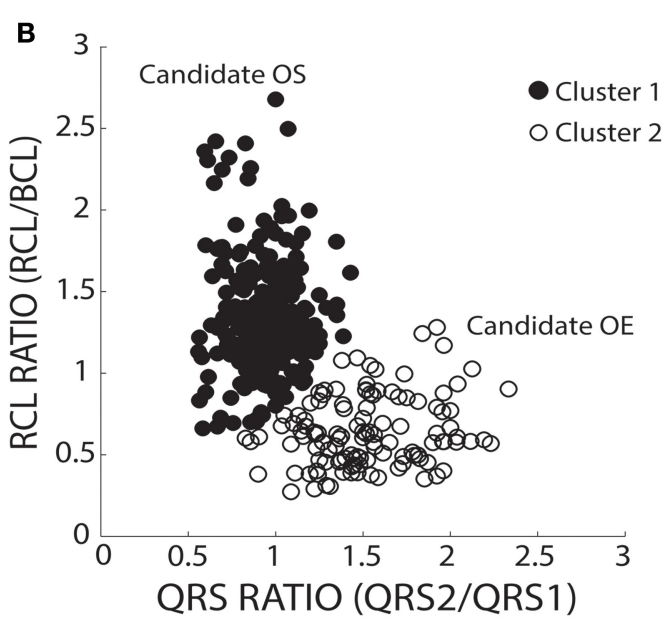

RCL and QRS ratios of all beats obtained from control, hypercalcemic, and digoxin interventions under both normal $\left(37^{\circ} \mathrm{C}\right)$ and hypothermic $\left(34^{\circ} \mathrm{C}\right)$ conditions for both slow (200 bpm) and fast ( $375 \mathrm{bpm})$ pacing rates. A k-means clustering algorithm was used to separate the data into 2 clusters. predominantly of beats with late relative RCL $(\leq 1)$ and narrow QRS complexes $(\sim 1)$, and Cluster 2 (open circles) is composed predominantly of beats with early RCL $(=1)$ and wide QRS complexes $(>1)$. The averages and standard deviations for these clusters are as follows: Cluster 1 RCL $1.31 \pm 0.33$ and QRS $0.94 \pm$ 0.16 and Cluster 2 RCL $0.64 \pm 0.22$ and QRS $1.53 \pm 0.32$. These Cluster 1 beats, therefore, may be overdrive suppressed, while Cluster 2 beats may be overdrive excited.

\section{CONTROL}

Keeping the cluster assignment for each recovery beat, we observed that control conditions only produced beats that were within Cluster 1 (Figure 5A, solid circles). Consistent with the theory of preferential overdrive suppression (OS) in normal hearts, faster pacing rates (gray circles) prolonged RCL relative to slower rates (black circles). For all experiments, rapid pacing at $375 \mathrm{bpm}$ significantly increased the RCL ratio without affecting QRS duration. Furthermore, mean native and recovery QRS durations from control hearts was $29 \pm 4 \mathrm{~ms}$, which is comparable to in vivo and ex vivo guinea pig measurements with atria intact (Stark et al., 1993; Batey et al., 1997). Therefore, these data suggest that recovery beats in these experiments with atria removed were all OS, and the pacemaker site for OS beats may be localized to the atrioventricular node or His bundles.

\section{CONTROL + HYPOTHERMIA}

Some studies have investigated spontaneous calcium release dynamics under hypothermic conditions (Wasserstrom et al., 2010; Plummer et al., 2011), which may impact TA characteristics. Therefore, we assessed recovery beats under physiologic hypothermia $\left(34^{\circ} \mathrm{C}\right)$. Consistent with control conditions, hypothermia only produced beats assigned to Cluster 1 (Figure 5B). Furthermore, RCL ratio was increased following faster relative to slower pacing (right panel, gray and black bars), while QRS ratio remained unchanged. As in control hearts, this is consistent with OS behavior. Lastly, hypothermia did not affect RCL or QRS ratios relative to control at normothermic conditions.

\section{HYPERCALCEMIA}

Hypercalcemia has previously been shown to induce spontaneous calcium releases and TA (Wasserstrom et al., 2010; Plummer et al., 2011). Hypercalcemia (5.5 mM) under normal physiological temperature was investigated, and surprisingly, produced beats preferentially assigned to Cluster 1 (Figure 6A, closed circles). Within Cluster 1, RCL and QRS ratios were unaffected by pacing rate. Due to lack of power, Cluster 2 data is presented but not statistically compared to Cluster 1.

\section{HYPERCALCEMIA + HYPOTHERMIA}

Slower pacing in hypercalcemic + hypothermic hearts resulted in more recovery beats classified in Cluster 1 (Figure 6B, black filled circles). During rapid pacing, more hearts exhibited Cluster 2 beats (gray open circles), and the RCL ratio of Cluster 2 beats was significantly shorter and QRS ratio significantly wider than Cluster 1 beats. Thus, overdrive excited (OE) beats were initiated under hypercalcemia during hypothermia. Surprisingly, the RCL ratio of Cluster 1 beats was significantly shorter at faster relative to slower pacing rates (right panel, closed bars). Hypothermia significantly decreased RCL ratio for fast paced Cluster 1 beats relative to normothermia in Figure 7A (\#).

\section{DIGOXIN}

Digoxin produced beats classified in Clusters 1 (closed circles) and 2 (open circles, Figure 7A). Like hypercalcemia, faster pacing did not increase RCL ratio for Cluster 1 beats as evidenced by black and gray filled circle overlap in the left panel and no difference between the average RCL ratios.

At faster pacing rates (Figure 7A, gray symbols), RCL ratio was significantly shorter for Cluster 2 relative to Cluster 1 and this 


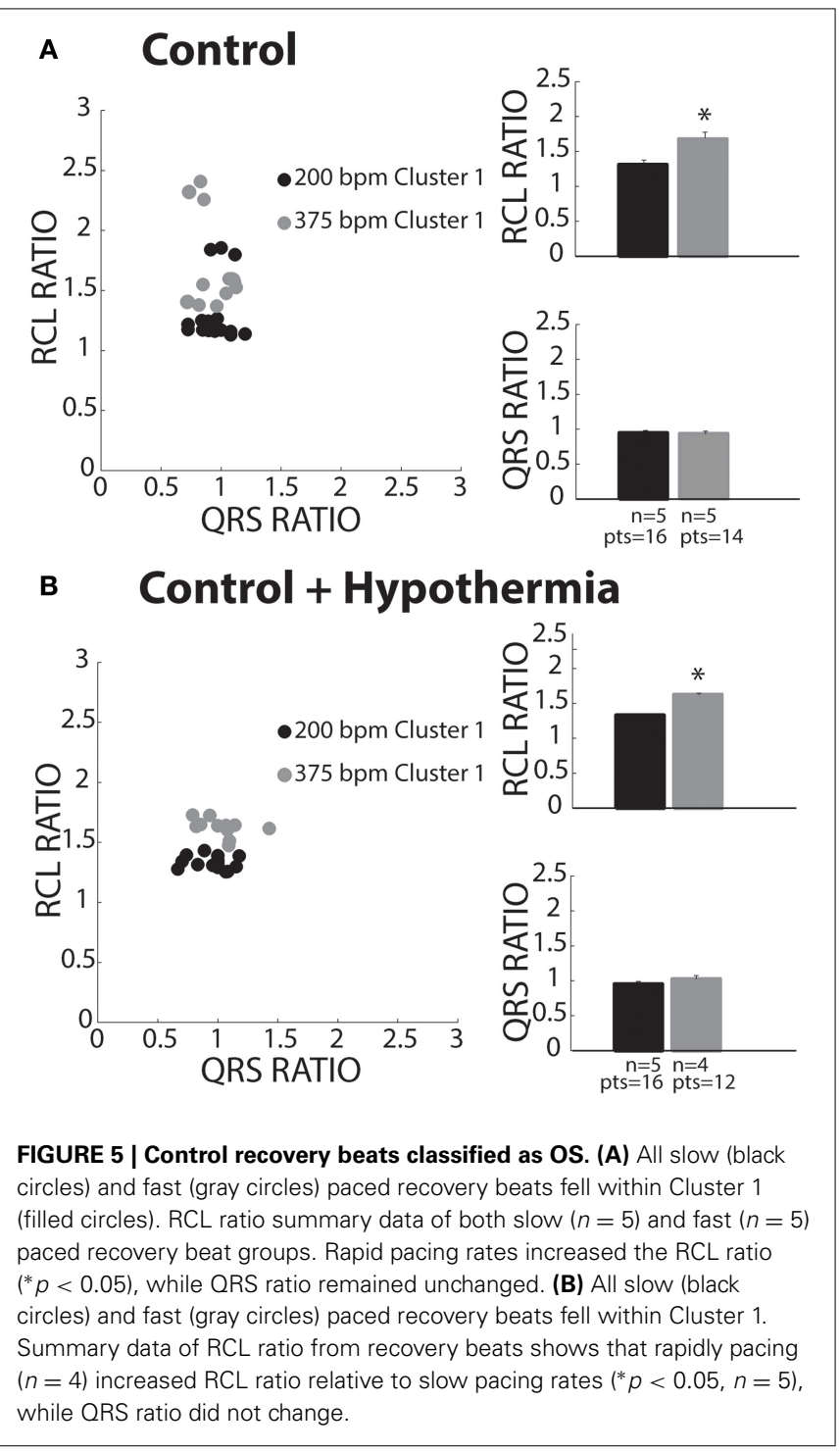

was paralleled by a significant increase in the QRS ratio. Cluster 2 , therefore, behaved as expected of OE recovery beats.

\section{DIGOXIN + HYPOTHERMIA}

Digoxin during hypothermia produced beats within Clusters 1 and 2 . The RCL ratio for Cluster 1 beats was not significantly affected by rapid pacing, suggesting that Cluster 1 beats were insensitive to overdrive pacing (Figure 7B, filled black and gray bars). This is similar to observations in normothermic digoxin conditions.

Regardless of pacing rate, Cluster 2 beats (Figure 7B, open bars) manifested decreased RCL and increased QRS ratios relative to the Cluster 1 beats (filled bars). Importantly, increasing pacing rate shortened the RCL ratio of Cluster 2 beats, as evidenced by the comparison of open bars (+, black and gray) without changing QRS ratio. Thus, Cluster 2 beats behaved as expected of OE beats.

With respect to normothermic digoxin in Figure 7A, hypothermic digoxin increased RCL ratio for Cluster 1 beats

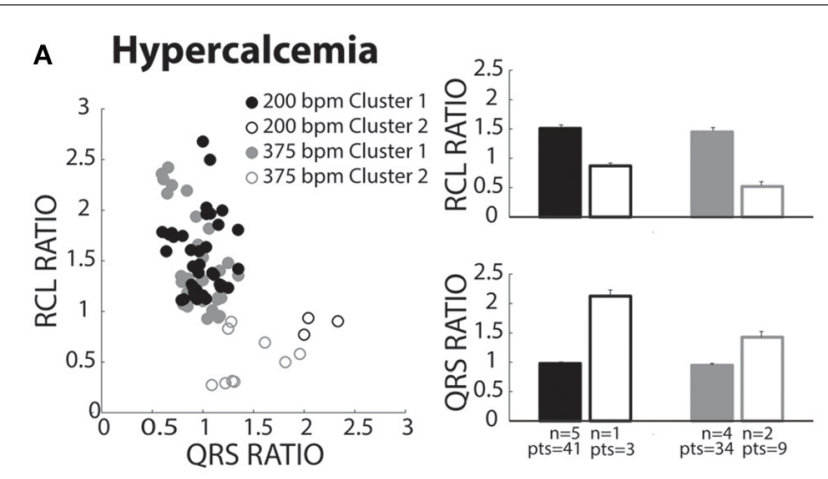

B Hypercalcemia + Hypothermia

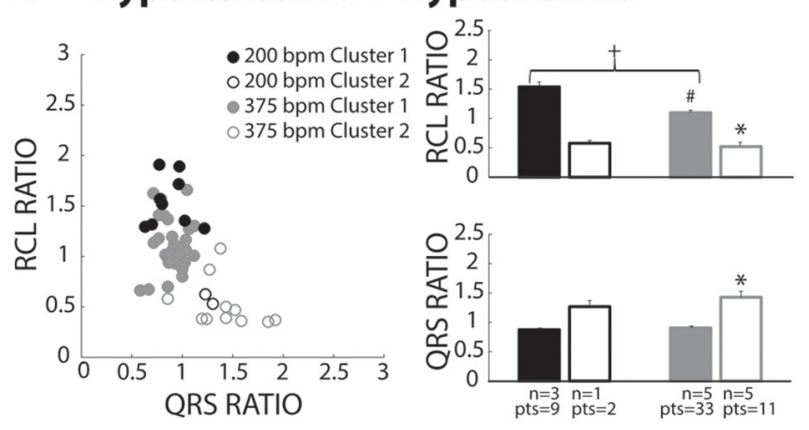

FIGURE 6 | Hypercalcemia recovery beats classified as both OS and OE. (A) Both slow (black circles) and fast (gray circles) paced beats fell within both Clusters 1 (closed circles) and 2 (open circles). Beats within Cluster 2 for both the slow $(n=1)$ and fast $(n=2)$ paced groups were insufficiently powered for statistical analysis. For Cluster 1 beats, pacing rate did not significantly change RCL or QRS ratio. (B) Pacing at slow (black circles) and fast (gray circles) rates created beats classified in Cluster 1 (filled circles) and Cluster 2 (open circles). Statistical analyses could not be performed with the fast paced Cluster 1 group due to lack of power $(n=1)$. Rapidly paced Cluster 2 group $(n=5)$ had a smaller RCL ratio when compared to the rapidly paced Cluster 1 group $(n=5, * p<0.05)$. For Cluster 1 , rapid pacing $(n=5)$ decreased the $\mathrm{RCL}$ ratio and increased QRS ratio relative to the slow paced group $(n=3,+p<0.05)$. Hypercalcemia and hypothermia $(n=5)$ for the rapidly paced Cluster 1 group (solid gray bar) decreased the RCL ratio when compared to normal hypercalcemia (Figure 6A, $n=5, \# p<0.05$ ).

following relatively slower pacing (Figure 7B, \#). Additionally, hypothermia increased the QRS ratio of Cluster 2 beats relative to normothermic digoxin (\#).

\section{OVERDRIVE EXCITATION INCIDENCE}

We also investigated the average incidence of Cluster 2 beats following rapid pacing in order to determine which experimental conditions produced more OE beats. Figure 8A demonstrates that temperature (open and filled bars) did not significantly affect OE beat incidence per animal during digoxin or hypercalcemia (black and gray bars). Further, during hypothermia, digoxin produced significantly more OE beats than hypercalcemia.

\section{MIXED POPULATION EFFECTS}

Figure 1B suggests that digoxin decreases RCL, but Figure 7A suggests that the population of Cluster 2 beats increases at faster pacing rates. Specifically Cluster 2 beats at $200 \mathrm{bpm}$ account for 


\section{A Digoxin}

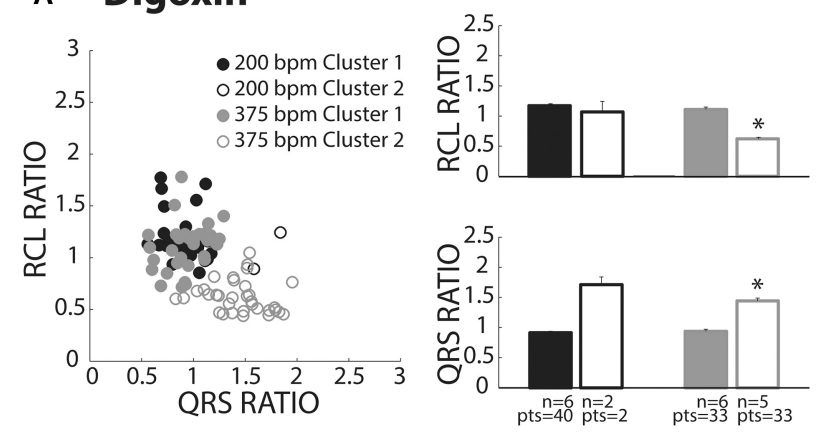

B Digoxin + Hypothermia
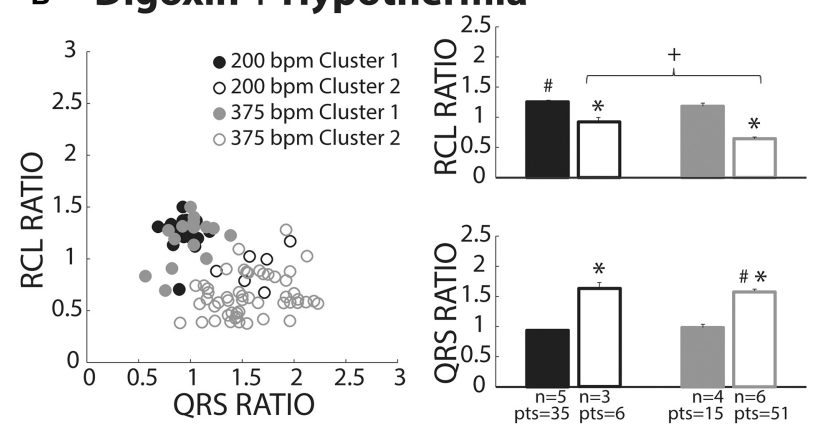

FIGURE 7 | Digoxin recovery beats classified as both OS and OE. (A) Recovery beats the result of slow (black circles) and fast (gray circles) pacing rates fell within both Clusters 1 (closed circles) and 2 (open circles). Statistical analyses could not be performed with the fast paced Cluster 1 group due to lack of power $(n=2)$. RCL ratio for the rapidly paced recovery beat group in Cluster $2(n=5)$ was shortened when compared to Cluster 1 ( $\left.n=6,{ }^{*} p<0.05\right)$. Rapidly paced recovery beats from Cluster 2 had a larger QRS ratio relative to the Cluster 1 group $\left({ }^{*} p<0.05\right)$. (B) Slow (black circles) and fast (gray circles) paced recovery beats fell within both Cluster 1 (filled circles) and Cluster 2 (open circles). Recovery beats the result of slow pacing in Cluster $2(n=3)$ had a smaller RCL ratio relative Cluster $1(n=5$, $\left.{ }^{*} p<0.05\right)$. The same occurred for rapidly paced Cluster $2(n=6)$ beats relative to Cluster $1\left(n=4,{ }^{*} p<0.05\right)$. Rapidly pacing the Cluster 2 group $(n=6)$ decreased the $\mathrm{RCL}$ ratio relative to the slow paced Cluster 2 group $(n=3,+p<0.05)$. Hypothermia also increased the RCL ratio for the slow paced Cluster 1 group $(n=5)$ relative to normal digoxin slow paced Cluster 1 group (Figure 7A, $n=6, \# p<0.05$ ). QRS ratio for the slow paced Cluster 2 group increased relative to Cluster $1(* p<0.05)$. The same occurred for the fast paced Cluster 2 group relative to Cluster $1\left({ }^{*} p<0.05\right)$.

Hypothermia increased the QRS ratio for the rapidly paced Cluster 2 group $(n=6)$ relative to normothermia $(\mathbf{A}, n=5, \# p<0.05)$.

2 out of 42 beats, while at $375 \mathrm{bpm}$ they account for 33 out of 66 beats. Thus, the decrease observed in Figure 1 may be a result of a switch at faster pacing rates from overdrive suppressed to overdrive excited beats. Figure 8B summarizes what happens to uncorrected RCL if Cluster 1 and 2 beats (gray and white respectively) are stratified from all recovery beats (black), and Figure 8C further demonstrates that the clustering algorithm accentuates the decrease in RCL ratio attributable to Cluster 2 beats. Furthermore, the RCL ratio demonstrates a similar relationship for stratified Cluster 2 beats, suggesting that the RCL ratio retains any relationship that RCL would have uncovered.

Stratifying between Cluster 1 and Cluster 2 beats also resulted in a much smaller variance for Cluster 2 beats for pacing rates greater than $250 \mathrm{bpm}$ (Figure 8D), suggesting that $\mathrm{OE}$ beats occurred within a narrow time window. However, these Cluster 2 beats did not manifest any significant changes in variance as a result of pacing rate, suggesting that these beats occurred within a narrow time window regardless of pacing rate.

\section{DIGOXIN + TETRODOTOXIN}

We sought to prospectively test the performance of this algorithm by utilizing a pharmacologic protocol previously demonstrated to reduce calcium mediated arrhythmia vulnerability (Rosen and Danilo, 1980; Radwanski et al., 2013). Hearts were perfused with digoxin and tetrodotoxin (TTX) and assigned to clusters based on the shortest distance to a priori cluster centroids determined from Figure 4. Hearts were only paced at $375 \mathrm{bpm}$ to preferentially elicit OE beats. As with digoxin, Cluster 2 beat (Figure 9A, open circles) RCL ratios were smaller $(0.73 \pm 0.04$ vs. $1.25 \pm 0.04)$ and QRS ratios larger ( $1.37 \pm 0.02$ vs. $1.02 \pm 0.02)$ relative to Cluster 1 beats. Importantly, while TTX did not change the QRS ratio for Cluster 2 beats, it significantly increased the RCL ratio (Figure 9B, gray bars).

A cross-correlation analysis was also performed to determine QRS morphology similarity between recovery beats and prepaced beats. The maximum cross-correlation coefficient from each analysis was determined for each recovery beat (Figure 9C). It was found that Cluster 2 beats had significantly smaller maximum correlation coefficients, indicating that QRS morphologies differed more from pre-paced beats. Stratified beat sensitivity and specificity were calculated using the maximum cross-correlation coefficient as the gold standard. Our clustering algorithm yielded high sensitivity (0.93) and specificity (0.94) relative to the cross-correlation algorithm (Table $\mathbf{1}$ ).

\section{DISCUSSION}

Rapid pacing is known to induce two types of recovery beats following the cessation of pacing: an interval of quiescence greater than or equal to the native rhythm, or a shortened interval. While we recapitulated previous studies demonstrating that RCL is proportional to pacing rate under control conditions (Malfatto et al., 1988; Iinuma et al., 1989) and inversely proportional during perfusion with a cardiac glycoside (Malfatto et al., 1988; Vos et al., 1990), the relationship observed for digoxin in Figure 1B may be misleading. More precisely, our beat classification system demonstrates that two distinct mechanistic populations compose recovery beats. The effect of grouping these mixed populations together would therefore significantly underestimate the magnitude of RCL shortening at very fast pacing rates. Therefore, in this study we present a methodology to differentiate between normal overdrive suppressed (OS) and arrhythmogenic excited (OE) rhythms independent of experimental intervention or interanimal variability.

Previous methods for analyzing triggered activity (TA) in whole hearts have included analyzing recovery beats: (1) of ventricular origin, (2) that have a shorter cycle length than the longest post-paced R-R interval, (3) with preceding delayed afterdepolarizations (DADs), or (4) with preceding spontaneous calcium releases (Malfatto et al., 1988; Furukawa et al., 1990; Vos et al., 1990; Plummer et al., 2011). The use of raw RCL and QRS 


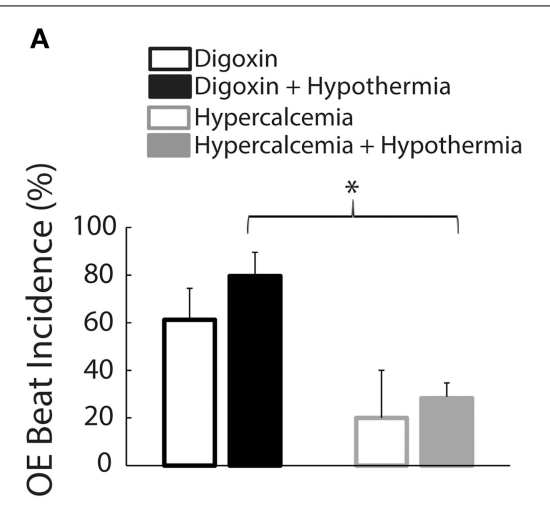

C

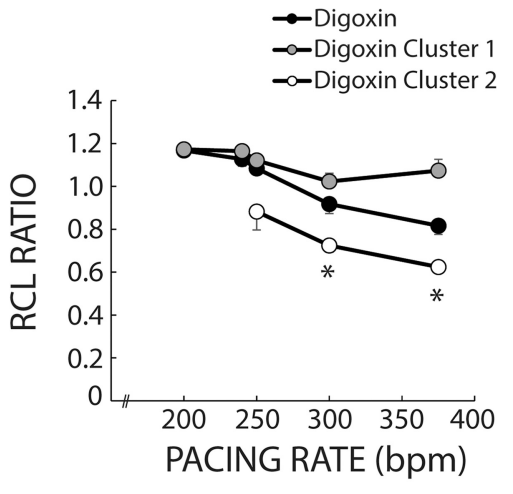

FIGURE 8 | Cluster separation reveals distinct OS and OE behaviors during Digoxin. (A) Average OE beat occurrence. Hypothermia did not significantly alter $\mathrm{OE}$ beat occurrence under either digoxin or hypercalcemic conditions. OE beat for hypothermic digoxin $(n=5)$ were significantly more frequent when compared to hypothermic hypercalcemia $(n=5, * p<0.05)$. (B) Recovery beat cycle lengths before stratification in black circles. After stratification there are significant difference between Clusters 1 (gray circles)

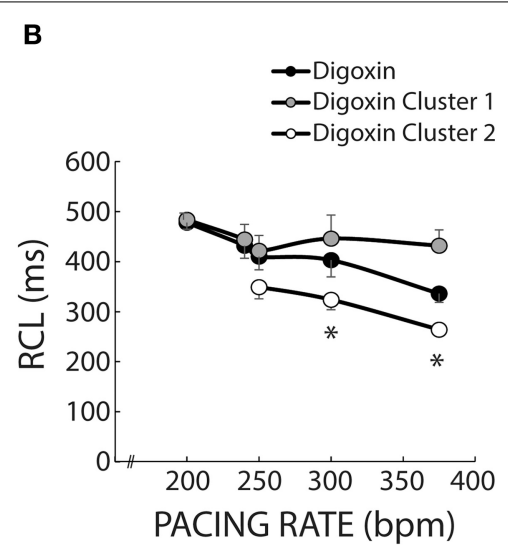

D

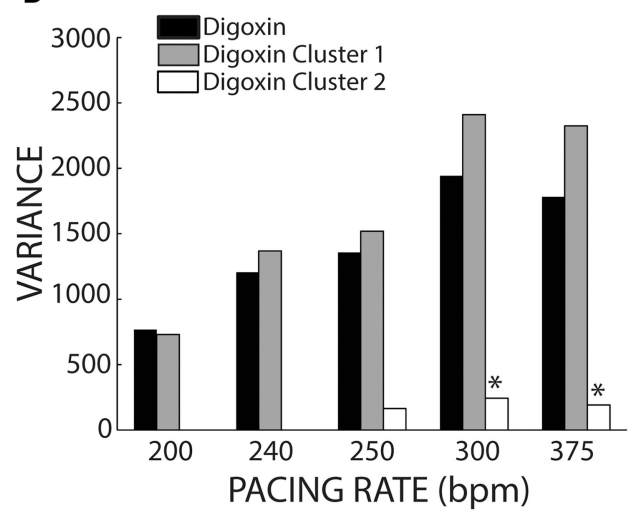

and 2 (white circles) at 300 and 375 bpm. Of note, only data with at least $n=3$ are plotted in panels (B-D). (C) RCL ratios for the entire dataset (black circles), Cluster 1 (gray circles), and Cluster 2 (white circles). Cluster 2 RCL ratios were significantly smaller when compared to Cluster 1 at 300 and $375 \mathrm{bpm}$. (D) Variance of the recovery beat cycle lengths for the entire digoxin data set (black), and Cluster 1 (gray) and 2 (white). Cluster 2 variance was significantly smaller than Cluster 1 at 300 and $375 \mathrm{bpm}$. values resulted in preferentially clustering based on RCL values. Moreover, native rhythms and QRS complexes were altered by certain interventions, thereby affecting the ability to identify normal and arrhythmogenic beats based on raw RCL and QRS values. Therefore, we sought a simple and robust method for differentiating between recovery beats.

Our methodology utilized both normalizing the recovery beat cycle length to the pre-paced beat cycle length and the recovery beat QRS duration to the pre-paced beat QRS duration. The finding that OE beat RCL ratios reduced in response to increased pacing rate is consistent with previous reports of similar measurements using normalized recovery beat cycle length to post-pacing R-R interval as a mechanistic stratifier (Vos et al., 1990). Indeed, RCL and RCL ratio for OE beats were significantly smaller when compared to OS beats, consistent with $\mathrm{OE}$ beat behavior. Additionally, the QRS normalization data suggests that $\mathrm{OE}$ beats may occur lower in the ventricular conduction system or ventricular tissue than OS beats, as has been previously suggested (Burashnikov and Antzelevitch, 2000).

\section{OVERDRIVE - SUPPRESSION - NORMOTHERMIA}

Our results demonstrated that control hearts only exhibited beats classified as OS, consistent with previous studies (Malfatto et al., 1988), and characteristic of normal automaticity. Other groups have proposed that OS may be caused by rapid pacing hyperpolarizing the membrane in response to activation of the sodium-potassium ATPase (NKA). (Vassalle, 1970; Abete and Vassalle, 1988) In contrast, the RCL ratio of OS beats during hypercalcemia was insensitive to pacing rate. To our knowledge, this is the first report of the effects of hypercalcemia on OS beats in whole heart preparations. Potential explanations for this finding may be that rapid pacing during hypercalcemia increases diastolic intracellular calcium more than under normocalcemic extracellular concentrations (Harding et al., 1993), thus augmenting NCX forward mode calcium extrusion, thereby increasing resting membrane potential (Hobai and O'Rourke, 2000), and perhaps making tissue hyperexcitable. Previous studies found that purkinje fibers recover more quickly from overdrive-induced hyperpolarization (Musso and Vassalle, 1982), or have an increased rate of diastolic depolarization 


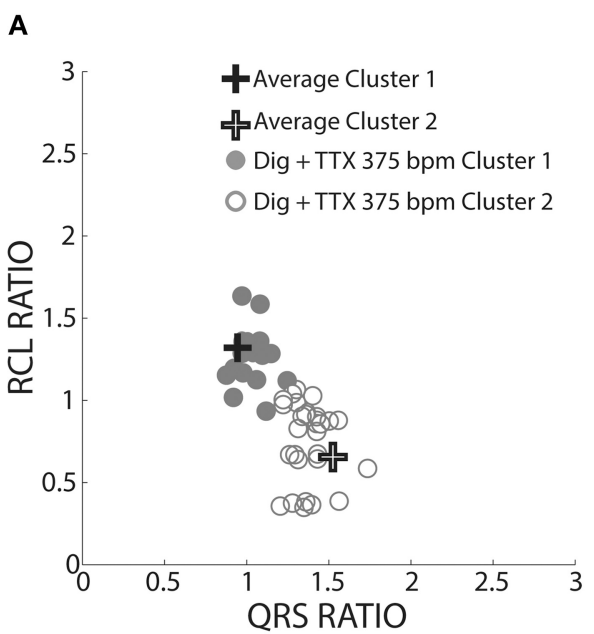

FIGURE 9 | TTX prolongs RCL without altering QRS of recovery beats. (A) Centroids of Clusters 1 and 2 derived from Figure $\mathbf{4}$ are plotted as + . Each Digoxin + TTX beat was classified based on least-squares distance from the centroid in either Cluster 1 (gray filled circles) or 2 (gray open circles). (B) Cluster 2 data from digoxin and digoxin + TTX reveals only RCL ratio

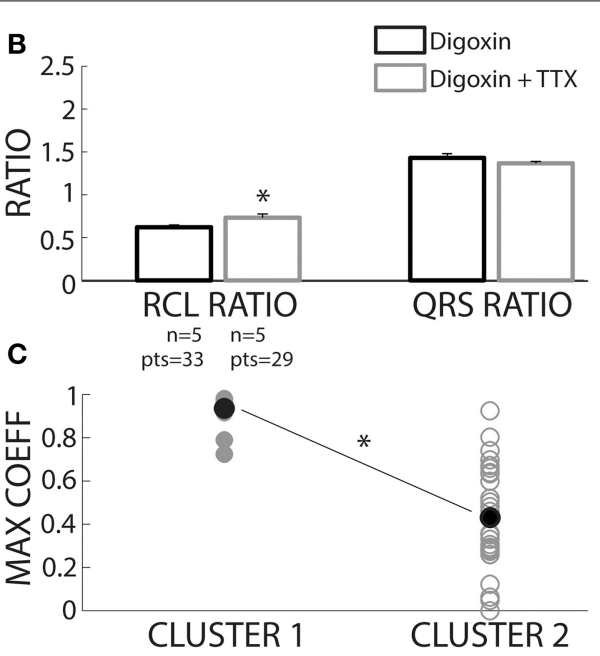

increased with TTX $(n=5, * p<0.05)$. (C) Cross-correlation analysis for QRS morphology reveals that Cluster 2 ( $n=5$ hearts, gray open circles) beat maximum correlation coefficients were significantly lower than Cluster 1 ( $n=4$ hearts, gray filled circles). Averages for each data set are shown as black filled circles.
Table 1 | Truth table: sensitivity and specificity of digoxin + TTX stratified beats.

\begin{tabular}{|c|c|c|c|}
\hline & & \multicolumn{2}{|c|}{ Cross-correlation } \\
\hline & & Ectopic beat & Normal beat \\
\hline \multirow{3}{*}{ 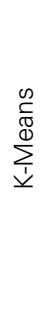 } & $\begin{array}{l}\frac{N}{\Phi} \\
\frac{ \pm}{0} \\
\frac{D}{U}\end{array}$ & True Positive: 28 & False Positive: 1 \\
\hline & $\begin{array}{l}\bar{c} \\
\bar{\Phi} \\
\frac{D}{0} \\
\frac{J}{U}\end{array}$ & False Negative: 2 & True Negative: 16 \\
\hline & & Sensitivity: 0.93 & Specificity: 0.94 \\
\hline
\end{tabular}

(Temte and Davis, 1967), when placed in high calcium solutions.

Directly inhibiting NKA with digoxin created OS beats insensitive to pacing rates. This is consistent with previous research that demonstrated cardiac glycosides can blunt the prolongation of the recovery interval typically seen under control conditions (Hogan et al., 1973). Furthermore, others have demonstrated that the glycoside ouabain abolishes overdrive hyperpolarization and shifts the maximum diastolic potential to more positive values in feline purkinje fibers (Browning et al., 1979), guinea pig and sheep ventricular muscle, and purkinje fibers (Cohen et al., 1982). In summary, the increase in RCL could be due to membrane hyperpolarization by NKA and, subsequently, NKA inhibition could blunt hyperpolarization and pacing rate-RCL dependence.

\section{OVERDRIVE - SUPPRESSION - HYPOTHERMIA}

The expected relationship between OS RCL and pacing rate is maintained for control conditions, but surprisingly increasing pacing rate during hypothermic hypercalcemia decreased OS RCL ratio. Sensitivity and specificity for the ratio analysis was high (0.8 and 0.98 , respectively) using a QRS cross-correlation analysis as the gold standard (Kyle et al., 2009). Furthermore, cross-correlation analysis revealed the same RCL to pacing rate relationship for OS beats. Both analyses suggest the surprising finding that rapid pacing may actually decrease the RCL of hypothermia + hypercalcemia OS recovery beats. As discussed above during normothermia, hypercalcemia may increase NCX activity and depolarize the membrane. Previous studies under severe hypothermia $\left(21-22^{\circ} \mathrm{C}\right)$ have suggested that NCX plays a larger role in calcium sequestration (Puglisi et al., 1996), which may augment membrane potential depolarization during rapid pacing, perhaps resulting in automatic recovery beats that return earlier. This hypothesis requires validation, however.

Lastly, OS RCL during hypothermic digoxin was insensitive to pacing rate. Unlike hypercalcemia which directly increases intracellular calcium by increasing the calcium driving force, NKA inhibition increases intracellular sodium prior to calcium. Therefore, NCX may not significantly depolarize the membrane during hypothermic digoxin and rapid pacing because intracellular sodium and calcium rise concurrently.

\section{OVERDRIVE - EXCITATION - NORMOTHERMIA}

Once again, control conditions never produced an OE beat in this study. In six hearts perfused with hypercalcemia, only two hearts produced OE beats and, therefore, conclusions cannot be readily drawn from this protocol. For digoxin, relatively slow pacing rates did not generate OE beats in sufficient hearts for statistical comparison or discussion.

Yet, RCL variance for digoxin OE beats was significantly smaller than for OS beats, suggesting that the mechanisms underlying $\mathrm{OE}$ beats may be more temporally deterministic than mechanisms underlying OS beats. Interestingly, RCL variance for 
OE beats did not change with pacing rate, which may be inconsistent with a previous study that demonstrated that increasing pacing rate in intact tissue decreases the standard deviation of spontaneous calcium release timing (Wasserstrom et al., 2010). Furthermore, post-hoc analysis of another study suggests that spontaneous calcium release timing variability in whole hearts decreases as a function of pacing rate as well (Plummer et al., 2011). This intriguing discrepancy could be a result of differences in spontaneous calcium releases and their subsequent fully propagated TA response.

\section{OVERDRIVE - EXCITATION - HYPOTHERMIA}

In contrast to normothermia, all five hearts perfused with hypothermic hypercalcemic solutions exhibited $\mathrm{OE}$ beats at the fastest pacing rates, but not at the slowest pacing rate, thus precluding a discussion of mechanisms. However, during hypothermic digoxin, the OE RCL ratio decreased as pacing rate increased, consistent with previous studies demonstrating that rapid pacing decreases RCL of TA (Furukawa et al., 1990; Plummer et al., 2011). The proposed mechanism of $\mathrm{OE}$ is due to a rise in diastolic intracellular calcium levels leading to increased probability of a spontaneous calcium release from the sarcoplasmic reticulum causing forward mode calcium extrusion through NCX thereby depolarizing the membrane prematurely (Wier and Beuckelmann, 1989; Schlotthauer and Bers, 2000). As mentioned previously, hypothermia can raise resting membrane potential and make tissue more likely to exhibit a triggered beat. In short, hypothermic digoxin not only produced the most OE beats, but the behavior of these beats was consistent with TA.

\section{SODIUM CHANNEL INHIBITION AND EXCITED BEATS}

We also investigated whether inhibition of TTX-sensitive sodium channels could impact the timing of OE beats. OE beat RCL ratio was prolonged in the presence of the sodium channel inhibitor tetrodotoxin (TTX). This is consistent with the finding that non-specific sodium channel blockers can delay the time to spontaneous calcium release (Lee et al., 2012). Indeed, previous studies have suggested that sodium channel inhibitors can act as antiarrhythmic agents, decreasing DAD amplitude and preventing OE beats (Rosen and Danilo, 1980; Radwanski et al., 2013). A few mechanisms have been proposed to explain the delay of onset of $\mathrm{OE}$ beats, such as decreased membrane excitability secondary to sodium channel inhibition. However, the native QRS duration and QRS ratio of OE beats remained unaffected by this degree of sodium channel inhibition, suggesting that membrane excitability was not altered. Further, we have shown that this concentration of TTX did not modify membrane excitability in an Andersen-Tawil syndrome guinea pig model of calcium mediated arrhythmias (Radwanski et al., 2013). Another possible mechanism is that TTX prevents intracellular calcium accumulation (Torres et al., 2010). While further investigation is needed to understand the mechanisms by which sodium channel inhibition affects $\mathrm{OE}$ beats, these results demonstrate that inhibiting sodium channels prolongs the time to the $\mathrm{OE}$.

\section{ALGORITHMIC PERFORMANCE}

The maximum coefficient from cross-correlation analysis of QRS complexes has previously been used to identify premature ectopic beats of ventricular origin, and resulted in high specificity and sensitivity values indicating that it is a good predictor for identifying ectopic beats (Kyle et al., 2009). Therefore, in this study, the lower maximum correlation coefficient found for $\mathrm{OE}$ beats suggests that our prospective analysis can distinguish between OS and OE beats. Furthermore, the sensitivity and specificity of our analysis validated against the maximum coefficient was high.

\section{CONCLUSIONS}

We present a new methodology for distinguishing between automatic and triggered activity. OS beats exhibited automatic behavior supported by data demonstrating that increasing pacing rate increased RCL ratio in control conditions. During hypothermic digoxin conditions $\mathrm{OE}$ beats manifested triggered activity characteristics as RCL ratio decreased. Moreover, OE beats manifested overdrive acceleration. These OE beats also exhibited larger QRS ratios and smaller RCL ratios when compared to OS beats within experimental conditions. Hypercalcemia and digoxin were able to create OE beats, whereas control conditions could not, supportive of the hypothesis that calcium overload underlies TA. Hypothermic digoxin produced the most OE beats. Sodium channel inhibition, which has been shown to ameliorate calcium mediated arrhythmia burden, increased the time to initiation of an $\mathrm{OE}$ beat. The prospective analysis performed well, suggesting that our methodology is able to distinguish between OS and OE beats. Our quantitative methodology presents a relatively simple technique for differentiating between automatic and triggered activity.

\section{LIMITATIONS}

The electromechanical uncoupler 2,3-diacetylmonoxime (BDM or DAM) was used in this study. This compound alters intracellular calcium concentrations and handling, and therefore may impact OE beat formation (Backx et al., 1994). Therefore, it may impact the occurrence of OE beats. Nevertheless, all experimental conditions were exposed to 2,3-diacetylmonoxime and thus its impact would have been included for all beats.

\section{DISCLOSURES}

The authors have submitted a University Invention Disclosure to Virginia Tech in regards to the quantitative methodology described in this study.

\section{ACKNOWLEDGMENTS}

A special thanks to Michael Heidinger at the Nora Eccles Harrison Cardiovascular Research and Training Institute at the University of Utah for his assistance in preliminary investigations.

\section{REFERENCES}

Abete, P., and Vassalle, M. (1988). Relation between Na+-K+ pump, Na+ activity and force in strophanthidin inotropy in sheep cardiac Purkinje fibres. J. Physiol. 404, 275-299. doi: 10.1113/jphysiol.1988.sp017290

Backx, P. H., Gao, W. D., Azan-Backx, M. D., and Marban, E. (1994). Mechanism of force inhibition by 2,3-butanedione monoxime in rat cardiac muscle: roles of [Ca2+]i and cross-bridge kinetics. J. Physiol. 476, 487-500. doi: 10.1113/jphysiol.1994.sp020149

Batey, A. J., Lightbown, I. D., Lambert, J. P., Edwards, G., and Coker, S. J. (1997). Comparison of the acute cardiotoxicity of the antimalarial drug halofantrine in vitro and in vivo in anaesthetize guinea-pigs. Br. J. Pharmacol. 122, 563-569. doi: 10.1038/sj.bjp.0701402 
Browning, D. J., Tiedeman, J. S., Stagg, A. L., Benditt, D. G., Scheinman, M. M., and Strauss, H. C. (1979). Aspects of rate-related hyperpolarization in feline Purkinje fibers. Circ. Res. 44, 612-624. doi: 10.1161/01.RES. 44.5.612

Burashnikov, A., and Antzelevitch, C. (2000). Block of I(Ks) does not induce early afterdepolarization activity but promotes beta-adrenergic agonist-induced delayed afterdepolarization activity. J. Cardiovasc. Electrophysiol. 11, 458-465. doi: 10.1111/j.1540-8167.2000.tb00342.x

Cohen, C. J., Fozzard, H. A., and Sheu, S. S. (1982). Increase in intracellular sodium ion activity during stimulation in mammalian cardiac muscle. Circ. Res. 50, 651-662. doi: 10.1161/01.RES.50.5.651

Cranefield, P. F. (1977). Action potentials, afterpotentials, and arrhythmias. Circ. Res. 41, 415-423. doi: 10.1161/01.RES.41.4.415

Ferrier, G. R., Saunders, J. H., and Mendez, C. (1973). A cellular mechanism for the generation of ventricular arrhythmias by acetylstrophanthidin. Circ. Res. 32, 600-609. doi: 10.1161/01.RES.32.5.600

Furukawa, T., Kimura, S., Catstellanos, A., Bassett, A. L., and Myerburg, R. J. (1990). In vivo induction of "focal" triggered ventricular arrhythmias and responses to overdrive pacing in the canine heart. Circulation 82, 549-559. doi: 10.1161/01.CIR.82.2.549

Harding, D. P., Smith, G. A., Metcalfe, J. C., Morris, P. G., and Kirschenlohr, H. L. (1993). Resting and end-diastolic [Ca2+]i measurements in the Langendorffperfused ferret heart loaded with a 19F NMR indicator. Magn. Reson. Med. 29, 605-615. doi: 10.1002/mrm.1910290505

Hashimoto, K., and Moe, G. K. (1973). Transient depolarizations induced by acetylstrophanthidin in specialized tissue of dog atrium and ventricle. Circ. Res. 32, 618-624. doi: 10.1161/01.RES.32.5.618

Hobai, I. A., and O'Rourke, B. (2000). Enhanced Ca (2+)-activated Na (+)-Ca (2+) exchange activity in canine pacing-induced heart failure. Circ. Res. 87, 690-698. doi: 10.1161/01.RES.87.8.690

Hogan, P. M., Wittenberg, S. M., and Klocke, F. J. (1973). Relationship of stimulation frequency to automaticity in the canine Purkinje fiber during ouabain administration. Circ. Res. 32, 377-384. doi: 10.1161/01.RES.32.3.377

Iinuma, H., Sekiguchi, A., and Kato, K. (1989). The response of digitalized canine ventricle to programmed stimulation: a study on triggered activity arrhythmias in the whole heart. Pacing Clin. Electrophysiol. 12, 1331-1346. doi: 10.1111/j.1540-8159.1989.tb05047.x

Kyle, A. M., Rogers, P. I., Han, S., Chen, P. S., and March, K. L. (2009). LifeShirt acquisition system to monitor ECG from ambulatory swine and the implementation of an arrhythmia detection algorithm. Conf. Proc. IEEE Eng. Med. Biol. Soc. 2009, 4820-4823. doi: 10.1109/IEMBS.2009.5332632

Lazzara, R., el-Sherif, N., and Scherlag, B. J. (1973). Electrophysiological properties of canine Purkinje cells in one-day-old myocardial infarction. Circ. Res. 33 722-734. doi: 10.1161/01.RES.33.6.722

Lee, Y. S., Maruyama, M., Chang, P. C., Park, H. W., Rhee, K. S., Hsieh, Y. C., et al. (2012). Ryanodine receptor inhibition potentiates the activity of $\mathrm{Na}$ channel blockers against spontaneous calcium elevations and delayed afterdepolarizations in Langendorff-perfused rabbit ventricles. Heart Rhythm. 9, 1125-1132. doi: 10.1016/j.hrthm.2012.02.031

Malfatto, G., Rosen, T. S., and Rosen, M. R. (1988). The response to overdrive pacing of triggered atrial and ventricular arrhythmias in the canine heart. Circulation 77, 1139-1148. doi: 10.1161/01.CIR.77.5.1139

Musso, E., and Vassalle, M. (1982). The role of calcium in overdrive suppression of canine cardiac Purkinje fibers. Circ. Res. 51, 167-180. doi: 10.1161/01.RES.51.2.167

Plummer, B. N., Cutler, M. J., Wan, X., and Laurita, K. R. (2011). Spontaneous calcium oscillations during diastole in the whole heart: the influence of ryanodine reception function and gap junction coupling. Am. J. Physiol. Heart Circ. Physiol. 300, H1822-H1828. doi: 10.1152/ajpheart.00766.2010
Priori, S. G., and Corr, P. B. (1990). Mechanisms underlying early and delayed afterdepolarizations induced by catecholamines. Am. J. Physiol. 258( $\mathrm{Pt} 2)$, H1796-H1805.

Puglisi, J. L., Bassani, R. A., Bassani, J. W., Amin, J. N., and Bers, D. M. (1996). Temperature and relative contributions of $\mathrm{Ca}$ transport systems in cardiac myocyte relaxation. Am. J. Physiol. 270(Pt 2), H1772-H1778.

Radwanski, P. B., Greer-Short, A., and Poelzing, S. (2013). Inhibition of Na+ channels ameliorates arrhythmias in a drug-induced model of Andersen-Tawil syndrome. Heart Rhythm. 10, 255-263. doi: 10.1016/j.hrthm.2012.10.005

Rosen, M. R., and Danilo, P. Jr. (1980). Effects of tetrodotoxin, lidocaine, verapamil, and AHR-2666 on Ouabain-induced delayed afterdepolarizations in canine Purkinje fibers. Circ. Res. 46, 117-124. doi: 10.1161/01.RES.46.1.117

Rosen, M. R., Gelband, H., and Hoffman, B. F. (1973). Correlation between effects of ouabain on the canine electrocardiogram and transmembrane potentials of isolated Purkinje fibers. Circulation 47, 65-72. doi: 10.1161/01.CIR.47.1.65

Schlotthauer, K., and Bers, D. M. (2000). Sarcoplasmic reticulum Ca $(2+)$ release causes myocyte depolarization. Underlying mechanism and threshold for triggered action potentials. Circ. Res. 87, 774-780. doi: 10.1161/01.RES.87.9.774

Stark, G., Stark, U., Bachernegg, M., Kasper, K., Kickenweiz, E., and Decrinis, M. (1993). A comparison of the effects of adenosine and verapamil on the conduction and pacemaker system of isolated guinea pig hearts. Clin. Cardiol. 16, 859-862. doi: 10.1002/clc.4960161205

Temte, J. V., and Davis, L. D. (1967). Effect of calcium concentration on the transmembrane potentials of Purkinje fibers. Circ. Res. 20, 32-44. doi: 10.1161/01.RES.20.1.32

Torres, N. S., Larbig, R., Rock, A., Goldhaber, J. I., and Bridge, J. H. (2010). Na+ currents are required for efficient excitation-contraction coupling in rabbit ventricular myocytes: a possible contribution of neuronal $\mathrm{Na}+$ channels. J. Physiol. 588(Pt 21), 4249-4260. doi: 10.1113/jphysiol.2010.194688

Vassalle, M. (1970). Electrogenic suppression of automaticity in sheep and dog purkinje fibers. Circ. Res. 27, 361-377. doi: 10.1161/01.RES.27.3.361

Vos, M. A., Gorgels, A. P., de Wit, B., Drenth, J. P., van Deursen, R. T., Leunissen, J. D., et al. (1990). Premature escape beats. A model for triggered activity in the intact heart? Circulation 82, 213-224.

Wasserstrom, J. A., Shiferaw, Y., Chen, W., Ramakrishna, S., Patel, H., Kelly, J. E., et al. (2010). Variability in timing of spontaneous calcium release in the intact rat heart is determined by the time course of sarcoplasmic reticulum calcium load. Circ. Res. 107, 1117-1126. doi: 10.1161/CIRCRESAHA.110.229294

Wier, W. G., and Beuckelmann, D. J. (1989). Sodium-calcium exchange in mammalian heart: current-voltage relation and intracellular calcium concentration. Mol. Cell. Biochem. 89, 97-102. doi: 10.1007/BF00220759

Conflict of Interest Statement: The authors declare that the research was conducted in the absence of any commercial or financial relationships that could be construed as a potential conflict of interest.

Received: 25 November 2014; accepted: 09 January 2015; published online: 18 February 2015.

Citation: Greer-Short A and Poelzing S (2015) Distinguishing between overdrive excited and suppressed ventricular beats in guinea pig ventricular myocardium. Front. Physiol. 6:14. doi: 10.3389/fphys.2015.00014

This article was submitted to Cardiac Electrophysiology, a section of the journal Frontiers in Physiology.

Copyright (c) 2015 Greer-Short and Poelzing. This is an open-access article distributed under the terms of the Creative Commons Attribution License (CC BY). The use, distribution or reproduction in other forums is permitted, provided the original author(s) or licensor are credited and that the original publication in this journal is cited, in accordance with accepted academic practice. No use, distribution or reproduction is permitted which does not comply with these terms. 